University of Nebraska - Lincoln

DigitalCommons@University of Nebraska - Lincoln

\title{
Implementation and modeling of a femtosecond laser-activated streak camera
}

\author{
Omid Zandi \\ University of Nebraska-Lincoln, ozandi2@unl.edu \\ Kyle J. Wilkin \\ University of Nebraska-Lincoln, kyle.j.wilkin@gmail.com \\ Martin Centurion \\ University of Nebraska - Lincoln, martin.centurion@unl.edu
}

Follow this and additional works at: https://digitalcommons.unl.edu/physicscenturion

Part of the Atomic, Molecular and Optical Physics Commons

Zandi, Omid; Wilkin, Kyle J.; and Centurion, Martin, "Implementation and modeling of a femtosecond laseractivated streak camera" (2017). Martin Centurion Publications. 30.

https://digitalcommons.unl.edu/physicscenturion/30

This Article is brought to you for free and open access by the Research Papers in Physics and Astronomy at DigitalCommons@University of Nebraska - Lincoln. It has been accepted for inclusion in Martin Centurion Publications by an authorized administrator of DigitalCommons@University of Nebraska - Lincoln. 


\title{
Implementation and modeling of a femtosecond laser-activated streak camera
}

\author{
O. Zandi, K. J. Wilkin, and M. Centurion \\ Department of Physics and Astronomy, University of Nebraska-Lincoln, Lincoln, Nebraska 68588, USA
}

(Received 30 January 2017; accepted 24 May 2017; published online 8 June 2017)

\begin{abstract}
A laser-activated streak camera was built to measure the duration of femtosecond electron pulses. The streak velocity of the device is $1.89 \mathrm{mrad} / \mathrm{ps}$, which corresponds to a sensitivity of $34.9 \mathrm{fs} / \mathrm{pixels}$. The streak camera also measures changes in the relative time of arrival between the laser and electron pulses with a resolution of $70 \mathrm{fs}$ RMS. A full circuit analysis of the structure is presented to describe the streaking field and the general behavior of the device. We have developed a general mathematical model to analyze the streaked images. The model provides an accurate method to extract the pulse duration based on the changes of the electron beam profile when the streaking field is applied. Published by AIP Publishing. [http://dx.doi.org/10.1063/1.4985008]
\end{abstract}

\section{INTRODUCTION}

Table-top ultrafast sub-relativistic electron sources with energies in the range of $10-100 \mathrm{keV}$ can be used to investigate ultrafast dynamics at the atomic level in condensed matter and isolated molecules. ${ }^{1-3}$ The temporal characterization of the electron pulses is essential for performing pump-probe experiments. Here, we present a detailed theoretical and experimental characterization of the performance of a femtosecond streak camera similar to the one developed by Kassier et al., ${ }^{4}$ which has been implemented in our gas phase ultrafast electron diffraction (UED) setup.

One of the main challenges in reaching femtosecond resolution in ultrafast electron diffraction (UED) is temporal broadening of the electron pulses due to the space charge effect. For UED in the gas phase, the velocity mismatch between laser and electron pulses (which causes the relative delay between them to change significantly as they traverse the target) poses an additional challenge. A possible solution to limit the space charge broadening is to use compact electron guns, where the distance from the source to the target is only a few centimeters. For UED on condensed matter samples, a resolution on the order of 200 fs has been reached with compact guns. ${ }^{5-8}$ For gas phase experiments, the vacuum requirements of the gun have so far prevented the use of very compact guns, but the temporal resolution was improved from several picoseconds ${ }^{9}$ to 850 fs by reducing the source-to-target distance to $10 \mathrm{~cm}^{3}$

An alternative solution to reach femtosecond resolution is to accelerate the electrons to relativistic energies of a few megaelectronvolts (MeV), which reduces the Coulomb expansion due to the relativistic effects ${ }^{10}$ and almost completely eliminates the velocity mismatch between collinearly propagating laser and electron pulses. A temporal resolution of 220 fs has been recently achieved using $\mathrm{MeV}$ electrons on gas phase targets. ${ }^{11-13}$ Generating and using relativistic electron pulses requires significant infrastructure, which is not accessible in most university-scale laboratories. An alternative that allows for the use of the more compact table-top setup is to use a DC keV accelerator followed by pulse compression using static ${ }^{14}$ or RF fields. ${ }^{15,16}$ It has been shown that highly charged photo-emitted electron pulses can be compressed to femtosecond duration using an RF cavity buncher. ${ }^{15-17}$ These electron pulses have been used in tabletop setups to capture ultrafast induced dynamics in condensed matter. ${ }^{18,19}$ In all cases, it is essential to accurately measure the duration of the electron pulses on target, as well as the arrival time of the electron pulses relative to the laser pulses. Different methods have been proposed and developed to measure the duration of electron pulses using streaking fields. ${ }^{4,20-27}$ Earlier versions of streak cameras were developed to measure the duration of $\mathrm{x}$-ray pulses. ${ }^{28-40}$ This method worked by first converting the x-ray pulse to an electron pulse in a photoemission process. Then a time-varying streaking field was used to map the longitudinal profile into a deflection angle. Finally, the pulse duration was retrieved by deconvolving the original transverse beam profile from the streaked profile. Measuring the duration of femtosecond pulses requires a very fast changing electric field to provide sufficient resolution. These time-varying streaking fields have been generated using a microwave cavity, ${ }^{19}$ a laser standing wave, ${ }^{21-23}$ a discharging capacitor, ${ }^{4,24}$ a split ring resonator, ${ }^{25}$ and a terahertz resonator. $^{27}$

A laser-activated streak camera is susceptible to time of arrival jitter between the laser and electron pulses. If the signal is averaged over many shots, the streak camera measures the convolution of the electron pulse duration with the time of arrival jitter. This is the relevant parameter that determines the temporal resolution in most UED experiments, where the signal is accumulated over many shots. If operated in singleshot mode, a streak camera can independently measure both the pulse duration and the timing jitter.

We have followed the general method for constructing a fast streak camera developed in Ref. 4, which used a charged capacitor set in parallel to a gallium-arsenide (GaAs) photoswitch. The photo-switch is activated by a laser pulse to discharge the capacitor. The time-dependent electric field of the capacitor is used to map the longitudinal position of the 
electrons within the bunch into a deflection angle. A high voltage photo-switch was first used to improve the temporal resolution and signal-to-noise ratio (SNR) in streaking tubes. ${ }^{29}$ A $\mathrm{GaAs}^{30}$ photo-switch was then used to increase the amplitude of the streaking field and hence the temporal resolution. ${ }^{31}$

The streaking velocity of the streak camera is determined by the initial voltage in the capacitor, the frequency of the oscillating field, and the quality factor of the circuit. The maximum field that can be used in the capacitor is limited by the breakdown voltage of the switch, which can be increased by using high voltage pulses to charge the capacitor. A fast oscillation frequency can be obtained by minimizing the capacitance and the self-inductance of the circuit. A millimetersized capacitor with a sufficiently small gap between its plates can provide a fast streaking field, while minimizing the total size of the circuit reduces the self-inductance. The quality factor is limited mostly by the Ohmic contacts of the photo-switch.

In Sec. II of this manuscript, we describe the components of the streak camera and present a detailed circuit analysis. We show that the damped harmonic oscillator is a good model for streak cameras with high streak velocities where a sufficiently high number of carriers are generated in the switch by the trigger laser pulse. In Sec. III, we present a mathematical model to simulate operation of the streak camera. This model can be used to extract the pulse duration from the streaked and unstreaked electron beam profiles for any type of streak camera. In Sec. IV, we describe the experimental setup. In Sec. V, we extract the electron pulse duration from experimental data. We compare the results of our method and the conventional method for extracting the pulse duration from the measurements. In addition, we show that the streak camera can be used to monitor the timing drift between the electron pulses and the triggering laser pulses.

\section{STREAK CAMERA THEORY AND CHARACTERIZATION}

\section{A. Geometry and the structure of the streak camera}

Figure 1 shows the basic components of the device. A charged capacitor is set in parallel to a GaAs photo-switch that is activated by a laser pulse. The electric field across the capacitor plates undergoes a damped oscillation with a period that is much longer than the transit time of the electron pulse through the capacitor. The rate of change of the field experienced by the electrons is approximately linear, which allows us to map the longitudinal profile of the electron beam onto its transverse profile. The streaked electron pulses are detected by a phosphor screen that is imaged onto a CCD camera.

\section{B. Circuit analysis of the streaking device}

The streaking device comprises the capacitor and the GaAs switch that are connected in parallel. There is a $25 \mu \mathrm{m}$ diameter pinhole in front of the device to truncate the electron beam before going through the capacitor. The primary function of the pinhole is to increase the resolution of the measurement

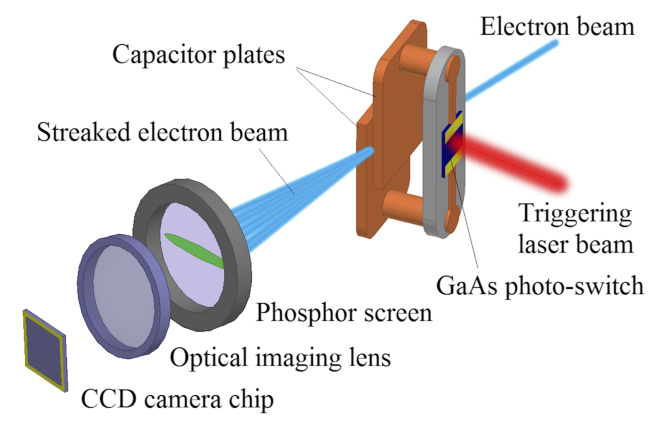

FIG. 1. Configuration of the streak camera. Once the GaAs photo-switch is activated by the laser pulse, the charged capacitor will be short-circuited. The damped oscillating field of the discharging capacitor will streak the electron pulse going through its plates. The streaked electron pulse hits a phosphor screen that is imaged onto a CCD camera by an optical lens.

by reducing the size of the electron beam on the screen. The schematic of the streaking device and its equivalent circuit are shown in Figs. 2(a) and 2(b), respectively. The capacitor has a capacitance $C$ and the complete circuit has a self-inductance $L$. The resistance of the circuit has two components: (i) the timeindependent resistor $R_{0}$ that stands for all the connections and is dominated by the Ohmic contacts of the photo-switch and (ii) the time-dependent resistor $R(t)$ that is the resistance of the photo-switch itself, which first decreases after the laser excitation and then increases due to electron-hole recombination. Figure 2(c) shows an electron deflected by the transverse electric field across the capacitor. The total time that an electron spends inside the capacitor is much shorter than the period of the electric field oscillation, so the electron displacement on the detector will depend on the instantaneous voltage across the capacitor $V_{C}(t)$. The full circuit analysis of the streaking device is provided in Appendix A. The displacement $x$ of an electron on the detector [see Fig. 2(c)], as a function of the arrival time of the triggering laser pulse $t$, satisfies the differential equation

$$
x(t)+L C \frac{d^{2} x(t)}{d t^{2}}+C \frac{d x(t)}{d t}\left(R_{0}+R(t)\right)=0 \quad(t \geq 0)
$$

for

$$
R(t)=R_{1} e^{\frac{t}{\tau}}
$$

and the initial conditions

$$
x(0)=x_{0}=\frac{e D s}{g m_{e} \gamma v_{z}^{2}} V_{0},\left.\quad \frac{d x}{d t}\right|_{t=0}=0 .
$$

In Eq. (1b), $R_{1}$ is the resistance of the photo-switch at $t=0$ which is the time when the laser pulse excites the photo-switch. The time constant $\tau$ depends on the structure of the switch and its Ohmic contacts. We performed an experiment to determine the value of $\tau$ (see Appendix A). In Eq. (1c), $e, m_{e}, v_{z}$, and $\gamma$ are the charge, mass, longitudinal velocity, and Lorentz factor of the electron, respectively. $s$ and $g$ are the length of the capacitor and the separation between its plates, respectively, and $D$ is the distance between the streaking device and the phosphor screen. The initial voltage of the capacitor is $V_{0}=V_{C}(0)$.

Figure 3 shows the electron pulse displacement on the detector as a function of the delay time. A solution to Eq. (1a) is fitted to the measurement points, where the fit parameters were the circuit self-inductance, the photo-switch contacts 


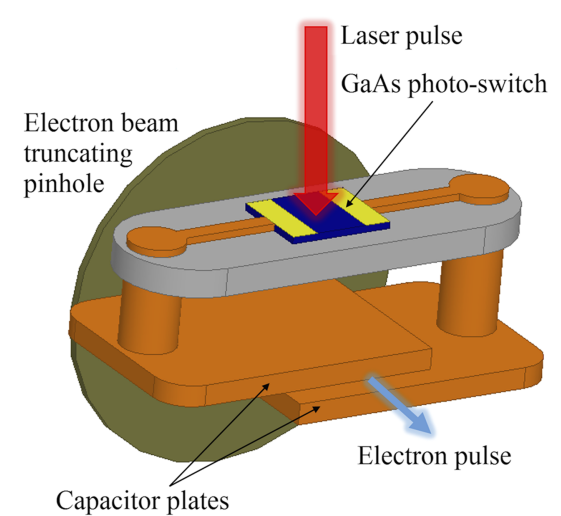

(a)

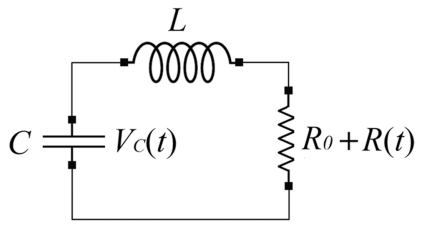

(b)
FIG. 2. (a) Basic illustration of the streaking device. (b) The equivalent circuit of the streaker while excited by the laser pulse. (c) The electron deflection by the capacitor electric field.

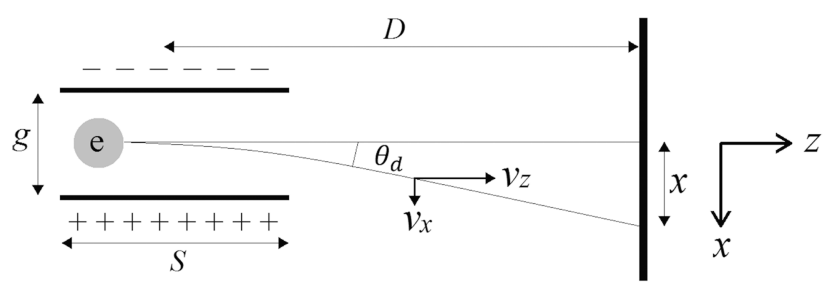

(c)

resistance, and the initial deflection point. The fitting allows evaluating the Ohmic contacts resistance of the photo-switch as well as an accurate value of the circuit self-inductance. For the case that the oscillation frequency of the device is larger than $1 / \tau$ and the trigger laser pulse energy is high enough to make $R_{1}$ much smaller than $R_{0}$, the time-dependent resistance of the photo-switch can be ignored in the first few oscillations because it is much smaller than the time-independent resistance of the Ohmic contacts. As a result, the streaking device can be modeled by a damped harmonic oscillator. The values we obtained for $\tau, R_{0}$, and $R_{1}$ indicate that we are well within that regime. In this case, the quality factor of the circuit is limited mostly by the Ohmic contacts of the photoswitch. A lower contact resistance increases the depth of the first minimum in Fig. 3 and improves the performance of the device.

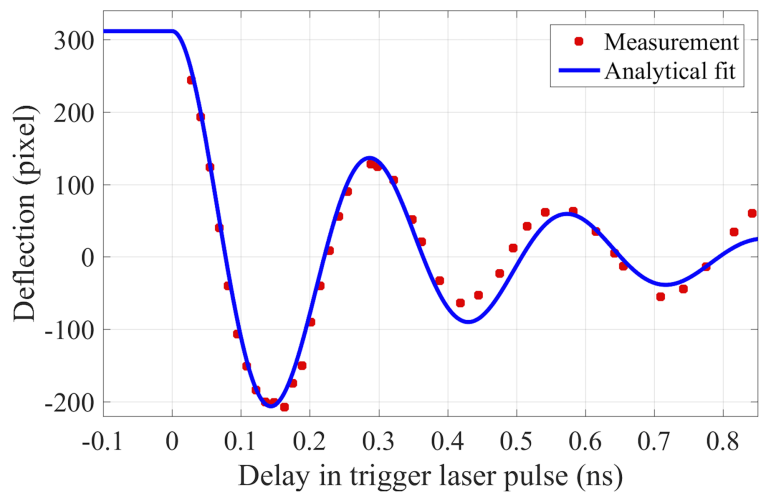

FIG. 3. Displacement of the electrons on the detector as a function of the trigger laser pulse arrival time (red dots) and the fitted function (blue curve). In this figure, $t=0$ is the moment when the laser pulse activates the switch, which was determined from the fit. In this experiment, we kept the initial voltage low $(350 \mathrm{~V})$ so that the maximum displacement during the oscillation could be captured on the detector.

\section{ELECTRON PULSE STREAKING PROCESS}

According to Eq. (A11) in Appendix A, the distance on the detector between two electrons with the same initial velocity going through the discharging capacitor at times $t$ and $t+\Delta t$ is

$$
\begin{aligned}
\Delta x & =x(t+\Delta t)-x(t)=\frac{e D s}{g m_{e} \gamma v_{z}^{2}} \frac{V_{C}(t+\Delta t)-V_{C}(t)}{\Delta t} \Delta t \\
& \approx \frac{e D s}{g m_{e} \gamma v_{z}^{2}} \frac{d V_{C}(t)}{d t} \Delta t .
\end{aligned}
$$

This distance is maximum when $\frac{d V_{C}(t)}{d t}$ is maximum. For this case, we write

$$
\Delta x=\kappa \Delta t,
$$

where

$$
\kappa=\left.\frac{e D s}{g m_{e} \gamma v_{z}^{2}} \frac{d V_{C}(t)}{d t}\right|_{\max }
$$

is the streak velocity. In literature, $\kappa$ is usually expressed in terms of angle of deflection per time and is the main parameter to describe the performance of the streaking device. However, $\kappa$ can alternatively be expressed in terms of pixels per time. In this case, $\kappa$ will describe the performance of the streak camera that is a combination of the parameters of the streaking device and the optical setup used to capture the image on the phosphor screen. We introduce a new quantity that reflects the sensitivity of the streak camera and can be measured directly,

$$
\varsigma=1 / \kappa .
$$

The value of $\varsigma$ was determined experimentally by measuring the electron beam displacement on the detector as a function of the time delay of the trigger laser around the time when $\frac{d V_{C}(t)}{d t}$ is maximum (at the first zero crossing of the capacitor electric field).

Consider an electron pulse with a negligible momentum spread traversing the capacitor plates such that the center of 
the pulse coincides with the first zero crossing of the capacitor voltage. If we set the origin of time at that moment, then an electron, entering the capacitor plates at the time $t$, will be deflected transversely by $x=\kappa t$. The electron pulses have a constant average longitudinal velocity $v_{z}$, and we can equivalently rewrite Eq. (2b) as

$$
x=\frac{\kappa}{v_{z}} z=\kappa^{\prime} z
$$

We call the dimensionless quantity $\kappa^{\prime}=\kappa / v_{z}$ the streak coefficient. Equation (4) means that an electron at point $z$, measured from the center of the electron pulse, will be deflected to point $x$ on the detector. Figure 4 illustrates this process.

Suppose the electron pulse is a uniformly charged spheroid with the transverse semi-axis $b$ and the longitudinal semi-axis $a$. This type of electron pulse can be recompressed in all directions by linear forces while retaining its spheroid profile. ${ }^{15}$ If we truncate this pulse by a pinhole of radius $\xi$ and then send it to the streaking device with streak coefficient $\kappa^{\prime}$, the intensity of the light emitted by the screen will be

$$
\begin{aligned}
I\left(x, y ; \kappa^{\prime}\right)= & \frac{\eta Q}{\frac{4}{3} \pi a b^{2}} \int_{-a}^{a} d z \Gamma\left(r(z)-\sqrt{\left(x-\kappa^{\prime} z\right)^{2}+y^{2}}\right) \\
& \times \Gamma\left(\xi-\sqrt{\left(x-\kappa^{\prime} z\right)^{2}+y^{2}}\right),
\end{aligned}
$$

provided the electron beam has no divergence between the streaking device and the detector. In Eq. (5), $Q$ is the charge of the electron pulse and $\eta$ is the quantum efficiency of the phosphor screen, i.e., the number of photons created by one electron hitting the phosphor screen. $\Gamma(x)$ is the Heaviside step function,

$$
\Gamma(x)=\left\{\begin{array}{ll}
1 & x>0 \\
0 & x<0
\end{array},\right.
$$

and $r(z)$ is the transverse radius of the uniformly charged spheroid,

$$
r(z)=b \sqrt{1-z^{2} / a^{2}} .
$$

Equation (7) can be replaced by any other electron pulse charge distribution and Eq. (5) can be modified accordingly.

In practice, the electron beam has some divergence, and also the phosphor screen has a finite resolution. We blur the

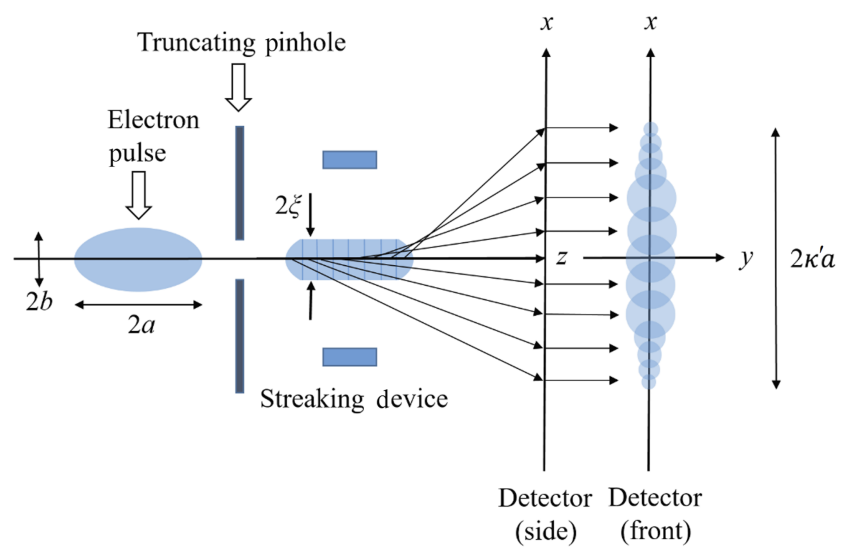

FIG. 4. Different sections of the electron pulse are deflected to different areas on the detector based on what time they pass through the streaking device. function in Eq. (5) by convolution with a Gaussian function to include the effect of these two parameters to give a blurred intensity

$I^{\text {blurred }}\left(x, y ; \kappa^{\prime}\right)=N \iint I\left(x^{\prime}, y^{\prime} ; \kappa^{\prime}\right) e^{-\frac{\left(x-x^{\prime}\right)^{2}+\left(y-y^{\prime}\right)^{2}}{2 \sigma^{2}}} d x^{\prime} d y^{\prime}$,

where $\sigma$ determines the width of the Gaussian blur and $N$ is a normalization constant such that

$$
\iint I^{\text {blurred }}\left(x, y ; \kappa^{\prime}\right) d x d y=\iint I\left(x, y ; \kappa^{\prime}\right) d x d y .
$$

This model is a general method and can be applied to any type of streak camera. In Appendix B, we provide more details on the mathematics of the model and evaluate the integral in Eq. (5) for both the unstreaked and streaked electron pulses to extract $a$ from the measurement data.

\section{EXPERIMENTAL SETUP}

The experimental setup is shown in Fig. 5. The electron pulses are generated by an ultraviolet femtosecond laser pulse in a 1-photon photoemission process and accelerated in a static electric field to a kinetic energy of $90 \mathrm{keV}$. The spatial profile of the UV beam on the photocathode is a truncated Gaussian, such that the photoemitted electron pulse will closely approximate a uniformly charged spheroid. ${ }^{41}$ These electron pulses can be reversibly recompressed in all directions by linear forces. We used the longitudinal time-dependent electric field of an RF cavity to temporally compress the electron pulses. ${ }^{15,16}$ The time-dependence of the electric field is such that opposing forces are applied to the back and front of the pulse. These forces result in electron pulses with a velocity distribution such that the pulses will self-compress at some distance after the RF cavity. The position of the minimum pulse duration depends on the amplitude of the applied electric field. The optimal compression is reached at the zero phase

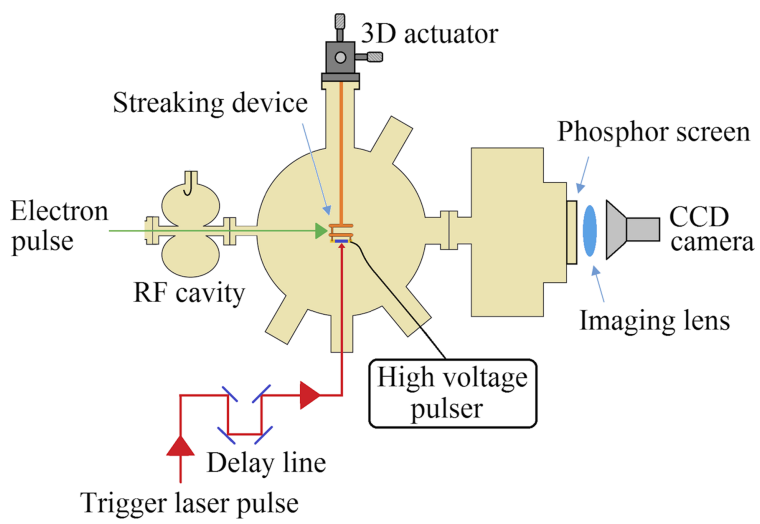

FIG. 5. Layout of the experiment. The photo-emitted electron pulses are compressed in time by the longitudinal component of an RF cavity electric field. The duration of these electron pulses are measured by the laser-activated streak camera explained in this study. The capacitor of the streaking device is fully charged by a high voltage pulse before the arrival of an electron pulse. The laser delay line is set so that the electrons traverse the capacitor around its electric field first zero crossing. 


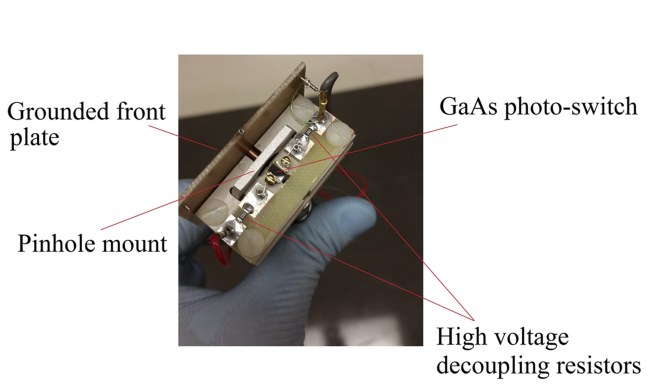

(a)

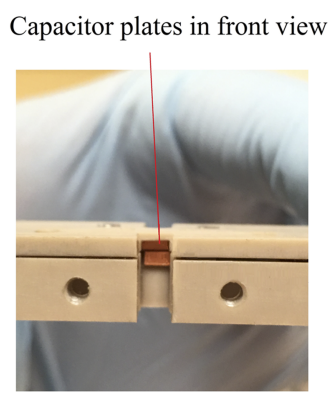

(b)
FIG. 6. Pictures of the streaking device (a) in the top view and (b) in the front view while the pinhole and the photo-switch were not assembled. of the longitudinal electric field of the RF cavity. The compression, then, will not change the average velocity of the electron pulse. However, in practice, the jitter in the RF cavity phase causes a jitter in the arrival time of the electron pulses. The jitter in the RF cavity amplitude will affect only the pulse duration. We used the streak camera to evaluate the temporal resolution of the experiment. In gas phase experiments, the scattering signal is gathered in an accumulation mode since the one-shot signal is not strong enough. Therefore, the temporal resolution of the experiment is a convolution of the actual pulse duration and the timing jitter over the measurement time.

\section{A. Streak camera parameters}

Figure 6 shows front and side view photographs of the streak camera. Table I lists the dimensions and other relevant parameters. The homemade GaAs photo-switch is composed of an undoped $5 \mathrm{~mm}$ wide GaAs wafer with two Ohmic contacts $2.4 \mathrm{~mm}$ apart on its sides. The contacts are composed of a $100 \mathrm{~nm}$ layer of gold on top of a $10 \mathrm{~nm}$ layer of chromium. The $3 \mathrm{~mm} \times 3 \mathrm{~mm}$ capacitor plates are $325 \mu \mathrm{m}$ apart resulting in a capacitance of $0.245 \mathrm{pF}$. The electron beam is truncated by a $25 \mu \mathrm{m}$ diameter pinhole before going through the capacitor plates. A $50 \mathrm{~ns}$ high voltage pulse initially charges the capacitor. The high voltage is pulsed to prevent damage to the GaAs photo-switch due to the discharge. This high voltage pulse is

TABLE I. Summary of the streaking device dimensions and parameters.

\begin{tabular}{lll}
\hline \hline Parameter & \multicolumn{1}{c}{ Description } & Value \\
\hline$l$ & Photo-switch Ohmic contacts separation & $2.4 \mathrm{~mm}$ \\
$W$ & Width of the photo-switch & $5 \mathrm{~mm}$ \\
$P_{0}$ & Laser power & $400 \mathrm{~mW}$ \\
$E_{0}$ & Laser pulse energy & $80 \mu \mathrm{J}$ \\
$R_{1}$ & Resistance of the photo-switch at t $=0$ & $0.372 \Omega$ \\
$\tau$ & Lifetime of the photo-switch & $0.36 \mathrm{~ns}$ \\
$R_{0}$ & Ohmic contacts resistance of the photo-switch & $48 \Omega$ \\
$s$ & Sides of the capacitor plates & $3 \mathrm{~mm}$ \\
$g$ & Separation between the capacitor plates & $325 \mu \mathrm{m}$ \\
$C$ & Capacitance of the streaking capacitor & $0.245 \mathrm{pF}$ \\
$L$ & Streaking device self-inductance & $8.35 \mathrm{nH}$ \\
$D$ & Distance between the streaking device & $57 \mathrm{~cm}$ \\
$\xi$ & and the detector & $12.5 \mu \mathrm{m}$ \\
\hline
\end{tabular}

supplied by a homemade device, with the schematic shown in Fig. 7. The pulser can provide $50 \mathrm{~ns}$ pulses with a repetition rate of $5 \mathrm{kHz}$ and voltages between 350 and $2000 \mathrm{~V}$. As shown in Fig. 6, there are two $2 \mathrm{k} \Omega$ resistors that decouple the oscillating voltage from the high voltage pulser and ground during the streak field oscillation. The value of the resistors is chosen so that in case of a laser pre-pulse arriving a few nanoseconds earlier, the streak capacitor can be recharged before the main laser pulse arrival. ${ }^{4}$ The capacitor electric field frequency of oscillation is $3.494 \mathrm{GHz}$, and the quality factor of its equivalent circuit is 3.85. Using the fit shown in Fig. 3, we determined the circuit self-inductance to be $L=8.35 \mathrm{nH}$ and the photo-switch contacts resistance $R_{0}=48 \Omega$. The resistance of the photo-switch at $t=0$ was calculated to be $R_{1}$ $=0.372 \Omega$, and the lifetime of the photo-switch was measured to be $\tau=0.36 \mathrm{~ns}$. The details of the calculation can be found in Appendix A.

The electrons were detected with a phosphor screen that was imaged onto a CCD camera with a pixel size of $9.9 \mu \mathrm{m}$. The demagnification of the imaging system is 3.74 , so each pixel corresponds to $37 \mu \mathrm{m}$ of the phosphor screen. The electron pulses are accelerated to $90 \mathrm{kV}$, corresponding to a gamma factor of $\gamma=1.176$. The calculated proportionality coefficient in Eq. (2b) is $\frac{e D s}{g m_{e} \gamma v_{z}^{2}}=32.5 \mu \mathrm{m} / \mathrm{V}$ equivalent to 0.88 pixels $/ \mathrm{V}$ for the imaging system. Figure 8 shows the position of the beam vs the corresponding delay in the trigger laser pulse for initial voltages $V_{0}=600 \mathrm{~V}, V_{0}=800 \mathrm{~V}$, and $V_{0}=1000 \mathrm{~V}$ around the first zero crossing of the capacitor electric field.

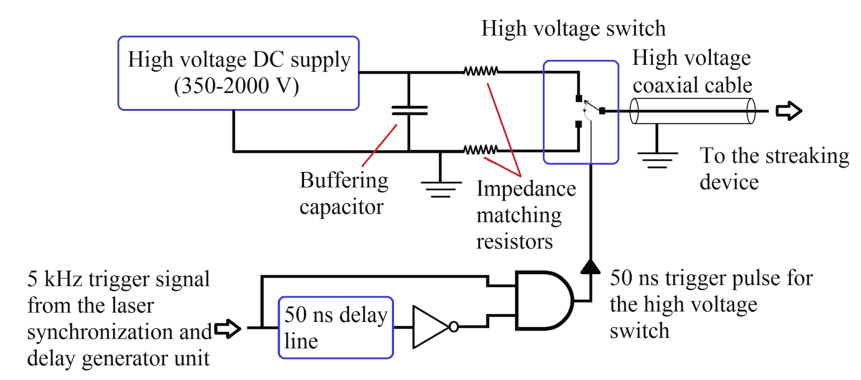

FIG. 7. Diagram for the pulser used to charge the streaking device. A high voltage DC supply is connected to the streaking device through a high voltage switch and a $75 \Omega$ coaxial cable. The high voltage switch is triggered by a $50 \mathrm{~ns}$ pulse which in turn is generated from a trigger signal coming from the laser synchronization and delay unit. There is a buffering capacitor between the supply and the switch to reduce the ripples in the high voltage pulse. Also there are two $75 \Omega$ resistors to match to the coaxial cable impedance to minimize the high voltage pulse reflection. 


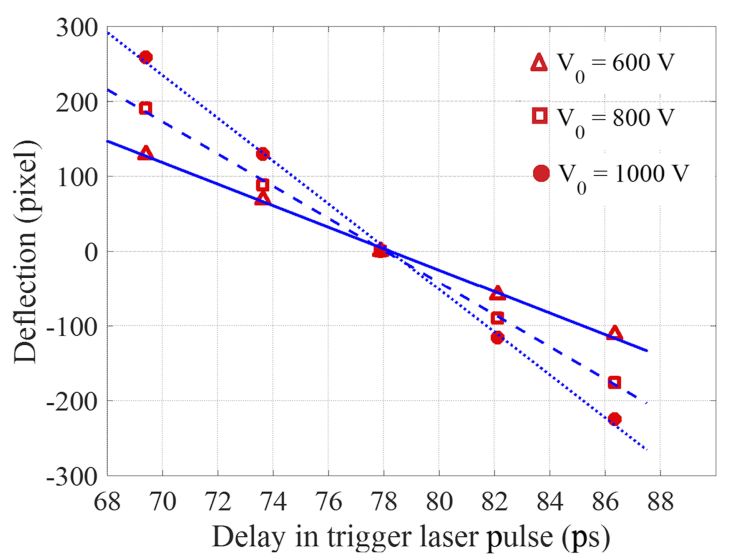

FIG. 8. Deflection of the electron beam as a function of the delay in the photo-switch trigger laser pulse. The red triangles, squares, and circles show the measured deflection as a function of the trigger delay around the first zero crossing of the capacitor electric field for initial voltages of $V_{0}=600 \mathrm{~V}, V_{0}$ $=800 \mathrm{~V}$, and $V_{0}=1000 \mathrm{~V}$, respectively. The corresponding values of $\varsigma$ 's are obtained by fitting lines to the data points and are $\varsigma=69.4 \mathrm{fs} /$ pixels, $\varsigma=46.5$ fs/pixels, and $s=34.9 \mathrm{fs} /$ pixels, respectively, equal to streak velocities of $1.89 \mathrm{mrad} / \mathrm{ps}, 1.4 \mathrm{mrad} / \mathrm{ps}$, and $0.94 \mathrm{mrad} / \mathrm{ps}$, respectively. These graphs can be considered the part of the oscillating graph shown in Fig. 2 around its first zero crossing for the higher initial voltages.

\section{B. Evaluation of timing drift}

Figure 9 shows the position of the electron beam on the detector around the first zero crossing of the capacitor electric field with the initial voltage of $600 \mathrm{~V}$ and trigger delay steps of 85 fs. The root-mean-square (RMS) error between the measurement and the fitted line is $70.3 \mathrm{fs}$. The fitted line has a slope of $65.3 \mathrm{fs} /$ pixels (equal to $1.75 \mathrm{mrad} / \mathrm{ps}$ streak velocity), which is close to what we had from Fig. 8 for $V_{0}$ $=600 \mathrm{~V}$. The measured fluctuations in the deflection can be attributed to the slow component of the timing jitter (timing drift) in the experiment. In this measurement, each data point is an average over ten consecutive frames each with an integration time of $500 \mathrm{~ms}$. In the current setup, the fast jitter cannot be measured because the detector requires an integration time of a few seconds to have a sufficient SNR. Accordingly, this

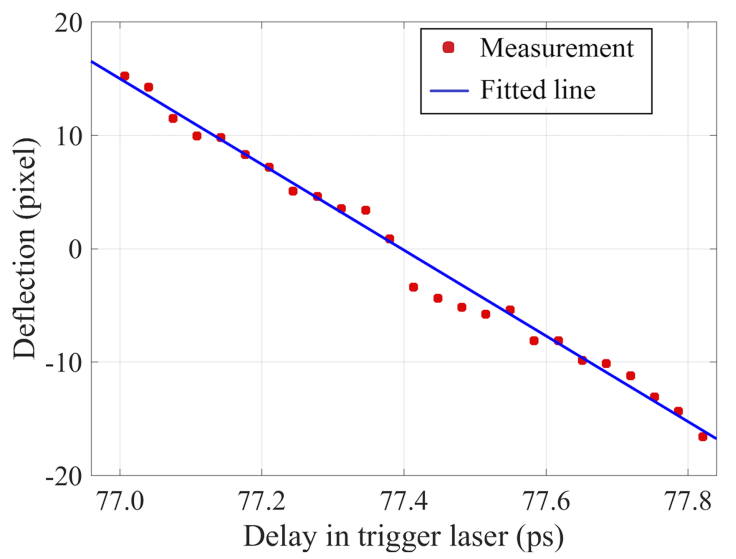

FIG. 9. Position of the electron beam center as a function of the trigger laser delay. The initial voltage of the capacitor is $600 \mathrm{~V}$. The trigger laser pulse was delayed in steps of $85 \mathrm{fs}$. This graph is the central part of the graph shown in Fig. 8 for $V_{0}=600 \mathrm{~V}$ and it shows the fluctuations in the arrival time of the electron pulses relative to the laser pulses. streak camera can be used to monitor changes in the arrival time of the electron pulses with respect to the trigger laser pulse down to around 70 fs RMS, with a measurement time of a few seconds. The measurement time could be reduced by using an image intensifier to enhance the signal from the phosphor screen or by increasing the number of electrons per pulse.

\section{PULSE DURATION MEASUREMENT RESULTS AND DISCUSSION}

Figure 10(a) shows an image of the streaked electron pulse composed of $5 \times 10^{5}$ electrons (before the pinhole at the entrance of the streak camera). In this figure, the electron pulse was not compressed and has a long streak length. Figure 10(b) shows the streaked pulse with the RF compression and Fig. 10(c) shows the compressed pulse with the streaking field off. Figure 10(d) shows the images corresponding to the compressed pulses integrated along the y direction. For this experiment, the electron beam was focused on the phosphor screen to minimize the spot size on the detector, which resulted in a fairly large beam (2.4 mm FWHM) on the streak camera. The beam can be made smaller on the target, but that results in a larger spot size on the detector which increases the uncertainty in the measurement.

We evaluated the pulse durations for the images in Fig. 10 by two methods. First, we used the conventional method of fitting Gaussian functions to the streaked and unstreaked pulses. If $\sigma_{s t}$ and $\sigma_{u s}$ are the standard deviations (STD) of the fitted Gaussians to the streaked and unstreaked pulses, respectively, the pulse duration can be obtained by the deconvolution,

$$
\Delta t_{S D T}=\varsigma \sqrt{\sigma_{s t}^{2}-\sigma_{u s}^{2}}
$$

where $\varsigma=69.4 \mathrm{fs} /$ pixels $=0.94 \mathrm{mrad} / \mathrm{ps}$. Figure $10(\mathrm{c})$ is the average of 10 measurements, which have resulted in a mean $\Delta t_{S D T}=206 \mathrm{fs}$, which when converted to a full width half maximum (FWHM) gives $\Delta t_{F W H M}=486 \mathrm{fs}$. The RMS deviation in the FWHM measurement over the 10 measurements was $4 \mathrm{fs}$. The streak camera works in the accumulative mode so the timing jitter in the GaAs photoswitch and the electron pulse arrival time are convolved into the measured value. The duration of the uncompressed pulse shown in Fig. 10(a) is $\Delta t_{S D T}=8.8$ ps or $\Delta t_{F W H M}$ $=20.7 \mathrm{ps}$.

The second method is based on the theory developed in Sec. III, assuming a spheroidal charge density distribution. The details of the calculations are provided in Appendix B. The pulse duration of the compressed electron pulses is $\Delta t_{s t d}=149$ fs equivalent to $\Delta t_{F W H M}=471 \mathrm{fs}$, with an RMS fluctuation of 4 fs over 10 measurements. The duration of the uncompressed pulses is $\Delta t_{\text {std }}=4.6 \mathrm{ps}$ equivalent to $\Delta t_{F W H M}=14.7 \mathrm{ps}$. Figures 11(a) and 11(b) show the results of the fitting for both methods.

For shorter pulse durations, the results of the two methods are similar. This is because the blurring due to the finite resolution of the detector and the divergence of the electron beam make the streaking trace approximately Gaussian when 


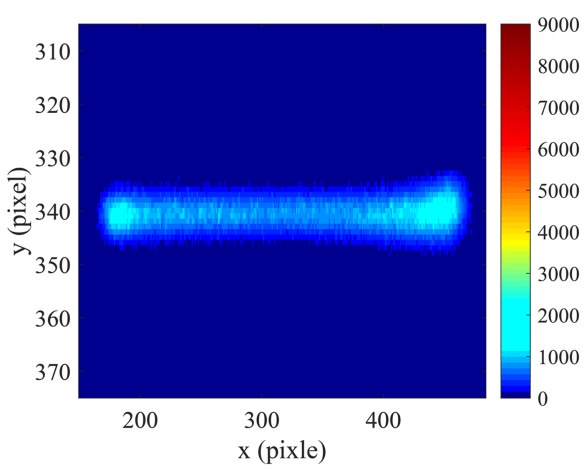

(a)

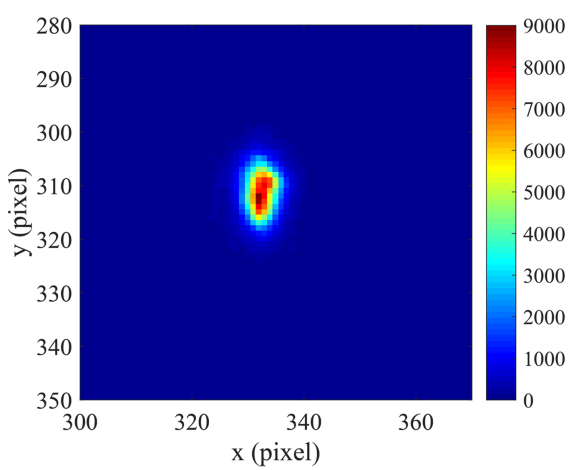

(c)

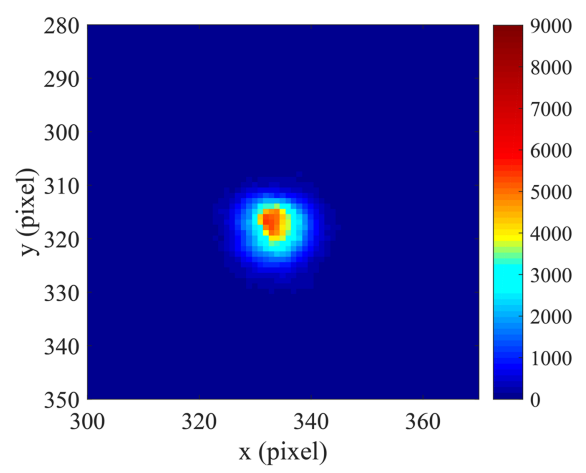

(b)

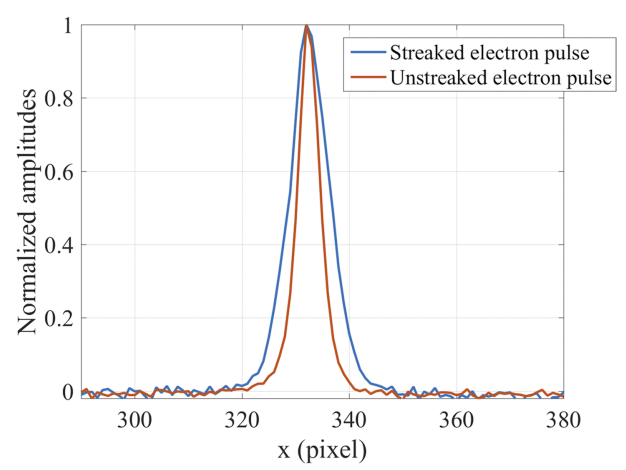

(d)
FIG. 10. (a) Image of the streaked uncompressed electron pulse; (b) image of the streaked compressed pulse; (c) image of the compressed unstreaked beam; (d) the streaked and unstreaked compressed pulses integrated over the $y$ axis. the initial beam width is comparable to the width after streaking. For longer pulse durations, the Gaussian method does not fit the data very well and will give inaccurate values for the pulse duration. The measured distribution in Fig. 11(b) also shows some difference with the fit, i.e., the maxima near the edges of the distribution. This difference is most likely caused by the fact that the electrons deflected at the larger angles will start to experience the fringe fields of the capacitor and be deflected less than they would if the field remained uniform.

Based on the measured performance, we expect that this streak camera will be able to measure pulse durations (convolved with the timing jitter) below $100 \mathrm{fs}$, although our electron gun at the moment cannot operate with a resolution below $400 \mathrm{fs}$, due mainly to timing jitter. Our device has a sensitivity of $\varsigma=34.9$ fs/pixels or a streak velocity of 1.89 $\mathrm{mrad} / \mathrm{ps}$ for $1000 \mathrm{~V}$ initial voltage with a typical beam size on the detector of 5 pixels FWHM. For a pulse duration of
$100 \mathrm{fs}$, the streaked image will have a width of about 6 pixels, which can be detected with good SNR in a measurement with an integration time of a few seconds. We have also considered the measurement timing jitter due to fluctuations in the photo-switch conduction band creation time, caused by fluctuations in the energy of the laser pulse trigger. This effect can be minimized by operating the switch in saturation (high laser fluence). We measured the change in the time of arrival of the electrons with respect to the zero crossing of the streaking electric field as a function of energy to be $12.5 \mathrm{fs} / \mu \mathrm{J}$ for a laser pulse energy of $80 \mu \mathrm{J}$ (fluence of $850 \mu \mathrm{J} / \mathrm{cm}^{2}$ ), which is consistent with previous results. ${ }^{24}$ The measured laser power fluctuation is $0.5 \%$, RMS, so the expected timing jitter in the photo-switch due to laser power fluctuations is less than 5 fs RMS. This makes the streak camera suitable for the electron pulse duration measurement for gas phase UED experiments where data are accumulated over many laser shots.

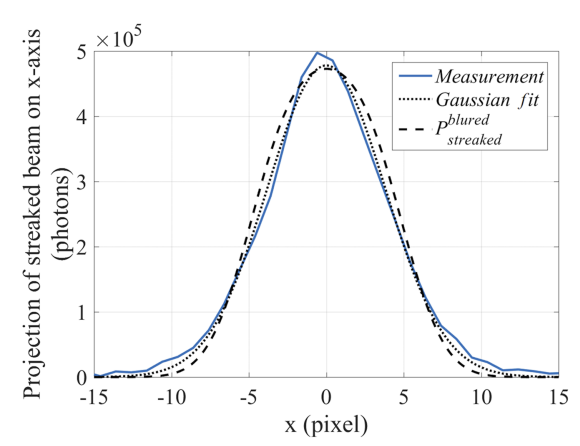

(a)

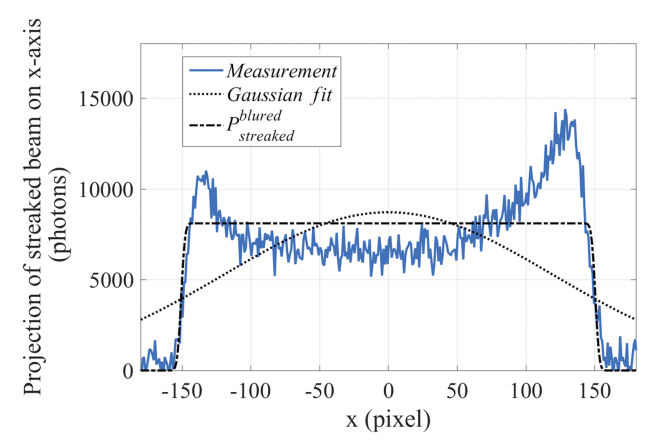

(b)
FIG. 11. (a) The compressed streaked pulse and (b) the uncompressed streaked pulse, both integrated over the $y$-axis together with the Gaussian function and the developed model fitted to them. See Appendix B for the definition of $P_{\text {streaked }}^{\text {blurred }}$. 


\section{CONCLUSION}

We constructed and characterized a femtosecond streak camera suitable for characterization of ultrafast electron pulses with a streak velocity of $1.89 \mathrm{mrad} / \mathrm{ps}$ for electrons accelerated to a kinetic energy of $90 \mathrm{kV}$. We provided a full circuit analysis of the streak camera and extracted all its equivalent circuit parameters to describe the oscillating field. The device produces a streaking field of $3 \mathrm{MV} / \mathrm{m}$ with an oscillation frequency of $3.49 \mathrm{GHz}$ and a quality factor of 3.85 . The quality factor was limited by the resistance of GaAs Ohmic contacts. We developed a general mathematical model that provides an accurate method to evaluate the pulse duration. We measured a pulse duration as low as 471 fs FWHM and showed that we can measure timing drifts that are as low as $70 \mathrm{fs}$ RMS. Based on the measured performance, we expect that the streak camera can be used to measure pulse durations below $100 \mathrm{fs}$. The performance could be further improved by increasing the voltage across the capacitor, reducing the size of the device to increase the discharge frequency and by improving the detection system to reduce the integration time. Using an image intensifier and a low noise detector could allow for single shot measurements.

\section{ACKNOWLEDGMENTS}

The authors would like to thank Gunther H. Kassier and Nicolas Erasmus for their advice and help on this project. This work was supported by the U.S. Air Force Office of Scientific Research, Ultrashort Pulse Laser-Matter Interactions program (Award No. FA9550-12-1-0149).

\section{APPENDIX A: CIRCUIT ANALYSIS OF THE STREAKING DEVICE}

The differential equation that describes the voltage across the capacitor after the laser excites the photo-switch is

$$
V_{C}(t)+L C \frac{d^{2} V_{C}(t)}{d t^{2}}+C \frac{d V_{C}(t)}{d t}\left(R_{0}+R(t)\right)=0,
$$

with the initial conditions

$$
V_{c}(0)=V_{0} ;\left.\frac{d V_{c}}{d t}\right|_{t=0}=0 .
$$

To solve Eq. (A1a) for $V_{C}(t)$, we need first to find the mathematical form of $R(t)$. The photon-created charge carrier density $n(t)$ can be estimated by ${ }^{42}$

$$
n(t)=\left(\frac{1-r}{V_{p}}\right) \frac{1}{E_{\lambda}} e^{-\frac{t}{\tau}} \int_{0}^{t} e^{\frac{t^{\prime}}{\tau}} P\left(t^{\prime}\right) d t^{\prime},
$$

where $r=0.325$ is the normal reflectance of the GaAs wafer for the $800 \mathrm{~nm}$ triggering laser pulse, ${ }^{43} E_{\lambda}=2.48 \times 10^{-19} \mathrm{~J}$ is the photon energy, and $P(t)$ is the laser power. $V_{p}=w l \delta_{d}$ is the photoconductive volume of the GaAs wafer with $w=5 \mathrm{~mm}$, $l=2.4 \mathrm{~mm}$, and $\delta_{d}=1 \mu \mathrm{m}$ being the width of the photoswitch, its length, and its $800 \mathrm{~nm}$ laser penetration depth, ${ }^{44}$ respectively. The time constant $\tau$ depends on the electron-hole recombination time $T_{r}$, the contact injection efficiency $\eta_{i}$, and the drift velocity $v_{d},{ }^{42}$

$$
\frac{1}{\tau}=\frac{1}{T_{r}}+\left(1-\eta_{i}\right) \frac{v_{d}}{l} .
$$

Equation (A2) assumes that the switch is illuminated uniformly, that the contact loss is equal for the electrons and holes, and that there is no carrier trapping. The laser pulse duration is assumed much shorter than the electron-hole recombination time in the GaAs photo-switch. Therefore, we approximate $P(t)$ by

$$
P(t)=E_{0} \delta(t),
$$

where $\delta(t)$ is the Dirac delta function, and $E_{0}=80 \mu \mathrm{J}$ is the laser pulse energy. By inserting Eq. (A4) into Eq. (A2), we get

$$
n(t)=\left(\frac{1-r}{V_{p}}\right) \frac{E_{0}}{E_{\lambda}} e^{-\frac{t}{\tau}}=n_{0} e^{-\frac{t}{\tau}} .
$$

By having $n(t)$, the photo-induced conductance of the wafer and hence its resistance are determined as

$$
\sigma(t)=\left(\mu_{e}+\mu_{h}\right) \text { en }(t)
$$

and

$$
R(t)=\frac{l}{w \delta_{d} \sigma(t)}=\frac{l^{2} E_{\lambda}}{\left(\mu_{e}+\mu_{h}\right) e(1-r) E_{0}} e^{\frac{t}{\tau}}=R_{1} e^{\frac{t}{\tau}},
$$

respectively, where $\mu_{e}=0.85 \mathrm{~m}^{2} \mathrm{~V}^{-1} \mathrm{~s}^{-1}$ and $\mu_{h}=0.04 \mathrm{~m}^{2}$ $\mathrm{V}^{-1} \mathrm{~s}^{-1}$ are the electron and hole mobilities, respectively, for the GaAs wafer. We found $R_{1}=0.372 \Omega$ using Eq. (A6b).

We find the time constant $\tau$ by exciting the photo-switch by a continuous wave (CW) laser at the same central wavelength of the pulsed laser. For the $\mathrm{CW}$ laser turned on at time $t=0, P(t)=P_{0} \Gamma(t)$, where $\Gamma(t)$ is the Heaviside step function in Eq. (6) and $P_{0}$ is the power of the laser. Therefore, from Eq. (A2), we find

$$
n(t)=\left(\frac{1-r}{V_{p}}\right) \frac{P_{0} \tau}{E_{\lambda}}\left[1-e^{-\frac{t}{\tau}}\right] .
$$

The steady-state photo-induced carrier density will be

$$
n_{s s}=n(t \rightarrow \infty)=\left(\frac{1-r}{V_{p}}\right) \frac{P_{0} \tau}{E_{\lambda}},
$$

and it results in the steady-state photo-switch resistance

$$
R_{s s}=\frac{l^{2} E_{\lambda}}{\left(\mu_{e}+\mu_{h}\right) e(1-r) P_{0} \tau}=\frac{\zeta_{s s}}{P_{0} \tau},
$$

where $\zeta_{s s}=1.4861 \times 10^{-5} \Omega \mathrm{J}$ is a constant. For low enough laser powers $P_{0}$ and recombination times of nanosecond order, $R_{S S}$ will be much larger than the contact resistance $R_{0}$. If we apply a constant voltage across the switch, by measuring the current going to the photo-switch as a function of the $\mathrm{CW}$ laser power, we can determine the time constant $\tau$. We used the circuit shown in Fig. 12(a) where the GaAs photo-switch was illuminated by a $\mathrm{CW}$ laser. The voltage measured by the voltmeter shown in Fig. 12(a) was used to determine $R_{s s}$ in Eq. (A8). Figure 12(b) shows $R_{s s}$ as a function of the laser 


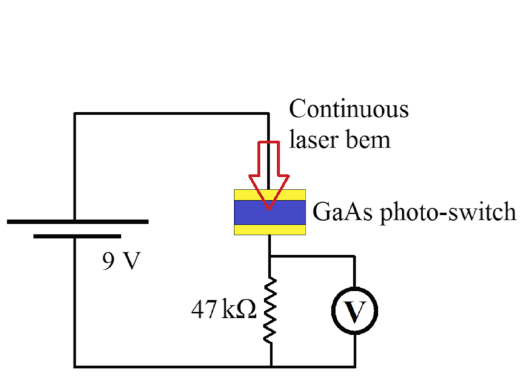

(a)

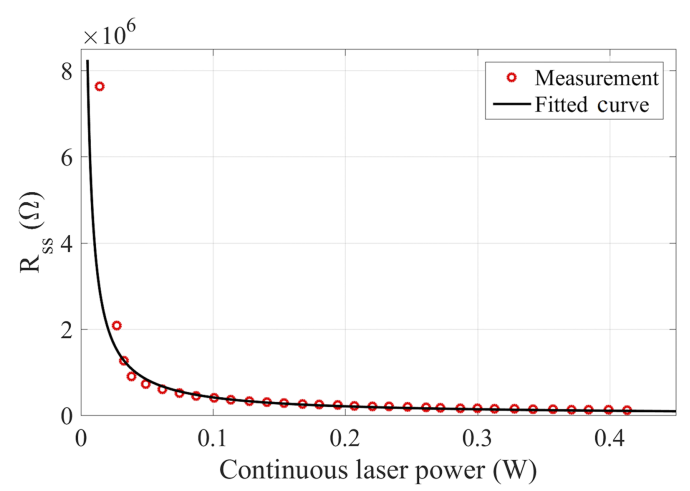

(b)
FIG. 12. (a) Circuit diagram of the setup used to measure the time constant $\tau$. A 9 V DC voltage supply is connected to the GaAs photo-switch that is in series with a $47 \mathrm{k} \Omega$ resistor. A voltmeter measures the voltage across this resistor. (b) The red spots are the resistance of the GaAs photo-switch obtained by recording the voltage across the $47 \mathrm{k} \Omega$ resistor in part (a) for different powers of the $\mathrm{CW}$ laser shining the GaAs photo-switch. The black curve is the fitted function in Eq. (A8). power as well as a fit to Eq. (A8) that gives a value of $\tau$ $=0.36 \mathrm{~ns}$.

The electron pulse travels between the plates of the capacitor and is deflected by the perpendicular electric field of the discharging capacitor. We record the position of the deflected electron pulse on the detector as a function of the delay between the electron pulse and the laser pulse that activates the GaAs photo-switch. The electron has an initial longitudinal velocity of $v_{z}$. The initial transverse velocity is zero. Suppose the capacitor electric field is in the $x$ direction. The electric field of the capacitor will cause the electron to gain a transverse velocity. The momentum gained in the $\mathrm{x}$ direction after traversing the capacitor is

$$
p_{x}=\int_{t}^{t+t_{s}} \frac{e V_{C}(t)}{g} d t \approx \frac{e V_{C}\left(t_{i n}\right)}{g} t_{s},
$$

where $t$ and $t_{s}=s / v_{z}$ are the time the electron enters the field region, and the amount of time it spends inside it, respectively. The tangent of the deflection angle $\theta_{d}$ will be

$$
\tan \theta_{d}=\frac{v_{x}}{v_{z}}=\frac{p_{x}}{p_{z}}=\frac{e V_{C}(t) t_{s}}{g m_{e} \gamma v_{z}} .
$$

For small deflection angles $v_{x} \ll v_{z}$, so from Eq. (A10), the displacement of the electrons on the detector will be

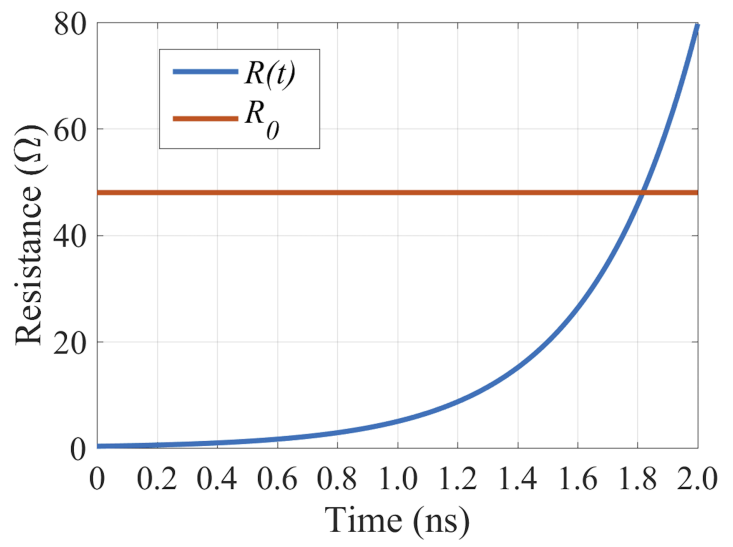

FIG. 13. Resistance of the GaAs switch (blue curve) compared with the invariant resistance of the rest of the device (red line). In this figure, $t=0$ is the time the laser hits the switch. The time-invariant resistance of the circuit is mostly due to the GaAs photo-switch contacts.

$$
x(t)=\frac{e D s}{g m_{e} \gamma v_{z}^{2}} V_{C}(\mathrm{t}) .
$$

The displacement $x(t)$ is linearly proportional to the voltage across the capacitor; therefore, it satisfies Eq. (1a).

As mentioned in Sec. II, the circuit constant resistance and self-inductance were found by best fitting a solution of Eq. (1a) to the measured electron displacement for different delays between the activating laser pulse and the electrons. We solved Eq. (1a) numerically by the finite difference method. In Fig. 13, the time-independent resistance of the circuit is compared with the time-dependent resistance of the photo-switch. As we see, the switch resistance is ignorable at least up to $1 \mathrm{~ns}$ after the excitation. If the streak camera electric field oscillates with a period smaller than $1 \mathrm{~ns}$, the effect of $R(t)$ is negligible and the streaking device can be modeled by a damped harmonic oscillator. However, for $R(t)$ to be small, the trigger laser pulse should have enough energy.

\section{APPENDIX B: MATHEMATICAL MODELING OF STREAK CAMERAS}

We photo-generate the electron pulses by ultrashort, truncated Gaussian transverse profile ultraviolet laser pulses. The electron pulses will evolve to uniformly charged spheroids. ${ }^{41}$ Therefore, here, we assume that the electron pulse is a uniformly charged spheroid with the longitudinal semi-axis $a$ and transverse semi-axis $b$. The electron pulse is truncated by a pinhole with a radius of $\xi$ before entering the streak camera. Each truncated pulse can be modelled as an infinite number of differential disks as shown in Fig. 4. Each differential disk of the electron pulse will be deflected at a different angle by the time-dependent streaking field. Ignoring the beam divergence between the streaking device and the screen, we can show from Eq. (5) for $\kappa^{\prime}=0$ that the detected intensity of the unstreaked pulse is

$$
I_{\text {unstreaked }}(x, y)=\frac{3 \eta Q}{2 \pi b^{2}} \sqrt{1-\frac{x^{2}+y^{2}}{b^{2}}} \Gamma\left(\xi-\sqrt{x^{2}+y^{2}}\right) .
$$

On the other hand, we can also show that the detected intensity of the streaked truncated pulse is 


$$
\left.\begin{array}{rl}
I_{\text {streaked }}\left(x, y ; \kappa^{\prime}\right)= & \frac{\eta Q}{\frac{4}{3} \pi a b^{2}} \times\left\{\left[\left(z-B^{-}\right) \Gamma\left(z-B^{-}\right)-\left(z-B^{+}\right) \Gamma\left(z-B^{+}\right)\right]_{-a}^{-a \sqrt{1-\frac{\xi^{2}}{b^{2}}}}+\left[\left(z-A^{-}\right) \Gamma\left(z-A^{-}\right)\right.\right. \\
& \left.-\left(z-A^{+}\right) \Gamma\left(z-A^{+}\right)\right]{\sqrt{1-\frac{\xi^{2}}{b^{2}}}}_{-a \sqrt{1-\frac{\xi^{2}}{b^{2}}}}^{a}\left[\left(z-B^{-}\right) \Gamma\left(z-B^{-}\right)-\left(z-B^{+}\right) \Gamma\left(z-B^{+}\right)\right]^{a} \\
a \sqrt{1-\frac{\xi^{2}}{b^{2}}}
\end{array}\right\} .
$$

Let $f(z)$ be any term appearing on the right hand side of Eq. (B2), and then we have $[f(z)]_{b}^{a}=f(a)-f(b)$. We also have

$$
\begin{aligned}
& A^{ \pm}=\frac{x}{\kappa^{\prime}} \pm \frac{\sqrt{\xi^{2}-y^{2}}}{\kappa^{\prime}} ; \\
& B^{ \pm}=\frac{\kappa^{\prime} x}{\kappa^{\prime 2}+\frac{b^{2}}{a^{2}}} \pm \sqrt{\frac{b^{2}-\left(x^{2}+y^{2}\right)}{\kappa^{\prime 2}+\frac{b^{2}}{a^{2}}}+\left(\frac{\kappa^{\prime} x}{\kappa^{\prime 2}+\frac{b^{2}}{a^{2}}}\right)^{2}} .
\end{aligned}
$$

We Gaussian blur the intensities in Eqs. (B1) and (B2) by the use of Eqs. (8) and (9) to include the divergence of the beams that comes from the initial transverse momentum spread and the space charge effect and also the broadening effect of the detector screen due to its finite resolution. Let $I^{\text {blured }}$ and $I_{\text {streaked }}^{\text {blured }}$ be the blurred versions of $I_{\text {unstreaked }}$ and $I_{\text {streaked }}$, respectively. The projection of $I_{\text {unstreaked }}^{\text {blured }}$ onto the X-axis is

$$
P_{\text {unstreaked }}^{\text {blured }}(x)=\int_{-\infty}^{\infty} I_{\text {unstreaked }}^{\text {blured }}(x, y) d y .
$$

We fitted $P_{\text {unstreaked }}^{\text {blured }}(x)$ to the corresponding measured data to find the width of the Gaussian blur in Eq. (8), i.e., $\sigma$. We also fitted $P_{\text {streaked }}^{\text {blured }}(x)$ calculated by

$$
P_{\text {streaked }}^{\text {blured }}\left(x ; \kappa^{\prime}\right)=\int_{-\infty}^{\infty} I_{\text {streaked }}^{\text {blured }}\left(x, y ; \kappa^{\prime}\right) d y
$$

to the corresponding measured streaked pulse to find the value of $a$. With the constraint

$$
\int_{-\infty}^{\infty} P_{\text {unstreaked }}^{\text {blured }}(x) d x=\int_{-\infty}^{\infty} P_{\text {streaked }}^{\text {blured }}\left(x ; \kappa^{\prime}\right) d x,
$$

we have only one degree of freedom that is $a$. The FWHM in this method is the FWHM of the function $A(z)=\pi r^{2}(z)$, for $r(z)$ in Eq. (7) and is equal to $\sqrt{2} a$. The standard deviation, on the other hand, is equal to $a / \sqrt{5}$.

The jitters in the photo-switch and the arrival time of electrons are convolved into the pulse duration we measured here. If we knew the value of those jitters, we could write

$I_{\text {streaked }}^{\text {blured }}(x, y)=N \iint I_{\text {streaked }}\left(x^{\prime}, y^{\prime}\right) e^{-\frac{\left(x-x^{\prime}\right)^{2}}{2\left(\sigma^{2}+\sigma_{\text {jitter }}^{2}\right)}-\frac{\left(y-y^{\prime}\right)^{2}}{2 \sigma^{2}}} d x^{\prime} d y^{\prime}$

instead of Eq. (9), while we should use Eq. (9) for the unstreaked pulse. In Eq. (B7), $\sigma_{\text {jitter }}$ is the standard deviation of the total jitter. By use of Eq. (B7), and knowing the value of $\sigma_{j i t t e r}$, we can extract the actual pulse duration by the method developed here.

${ }^{1}$ A. H. Zewail, Annu. Rev. Phys. Chem. 57, 65 (2006).

${ }^{2}$ G. Sciaini and R. J. D. Miller, Rep. Prog. Phys. 74, 096101 (2011).

${ }^{3}$ C. J. Hensley, J. Yang, and M. Centurion, Phys. Rev. Lett. 109, 133202 (2012).

${ }^{4}$ G. H. Kassier, K. Haupt, N. Erasmus, E. G. Rohwer, H. M. Von Bergmann, H. Schwoerer, S. M. Coelho, and F. D. Auret, Rev. Sci. Instrum. 81, 105103 (2010).

${ }^{5}$ B. J. Siwick, J. R. Dwyer, R. E. Jordan, and R. J. D. Miller, Science 302, $1382(2003)$

${ }^{6}$ R. Ernstorfer, M. Harb, C. T. Hebeisen, G. Sciaini, T. Dartigalongue, and R. J. D. Miller, Science 323, 1033 (2009).

${ }^{7}$ G. Sciaini et al., Nature 458, 56 (2009).

${ }^{8}$ P. Baum, D.-S. Yang, and A. H. Zewail, Science 318, 788 (2007).

${ }^{9}$ J. C. Williamson, J. Cao, H. Ihee, H. Frey, and A. H. Zewail, Nature 386(6621), 159 (1997).

${ }^{10}$ M. Reiser, Theory and Design of Charged Particle Beams (John Wiley \& Sons, 2008), Chap. 5.

${ }^{11}$ J. Yang, M. Guehr, T. Vecchione, M. S. Robinson, R. Li, N. Hartmann, X. Shen et al., Faraday Discuss. 194, 563 (2016).

${ }^{12}$ J. Yang, M. Guehr, T. Vecchione, M. S. Robinson, R. Li, N. Hartmann, X. Shen et al., Nat. Commun. 7, 11232 (2016).

${ }^{13}$ J. Yang, M. Guehr, X. Shen, R. Li, T. Vecchione, R. Coffee, J. Corbett et al., Phys. Rev. Lett. 117(15), 153002 (2016).

${ }^{14}$ Y. Wang and N. Gedik, IEEE J. Sel. Top. Quantum Electron. 18(1), 140-147 (2012).

${ }^{15}$ T. Van Oudheusden, E. F. De Jong, S. B. Van der Geer, W. P. E. M. Op't Root, O. J. Luiten, and B. J. Siwick, J. Appl. Phys. 102, 093501 (2007).

${ }^{16}$ T. Van Oudheusden, P. L. E. M. Pasmans, S. B. Van Der Geer, M. J. De Loos, M. J. Van Der Wiel, and O. J. Luiten, Phys. Rev. Lett. 105, 264801 (2010)

${ }^{17}$ G. H. Kassier, N. Erasmus, K. Haupt, I. Boshoff, R. Siegmund, S. M. M. Coelho, and H. Schwoerer, Appl. Phys. B 109, 249 (2012).

${ }^{18}$ V. R. Morrison, R. P. Chatelain, K. L. Tiwari, A. Hendaoui, A. Bruhács, M. Chaker, and B. J. Siwick, Science 346, 445 (2014).

${ }^{19}$ M. Gao, C. Lu, H. Jean-Ruel, L. C. Liu, A. Marx, K. Onda, S. Koshihara et al., Nature 496, 343 (2013).

${ }^{20}$ T. van Oudheusden, J. R. Nohlmans, W. S. C. Roelofs, W. P. E. M. Op't Root, and O. J. Luiten, Ultrafast Phenomena XVI (Springer Berlin Heidelberg, 2009), pp. 938-940.

${ }^{21}$ C. T. Hebeisen, R. Ernstorfer, M. Harb, T. Dartigalongue, R. E. Jordan, and R. J. D. Miller, Opt. Lett. 31, 3517 (2006).

${ }^{22}$ C. T. Hebeisen, G. Sciaini, M. Harb, R. Ernstorfer, T. Dartigalongue, S. G. Kruglik, and R. J. D. Miller, Opt. Express 16, 3334 (2008).

${ }^{23}$ M. Gao, H. Jean-Ruel, R. R. Cooney, J. Stampe, M. de Jong, M. Harb, G. Sciaini, G. Moriena, and R. J. D. Miller, Opt. Express 20, 12048 (2012).

${ }^{24}$ M. Gao, Y. Jiang, G. H. Kassier, and R. J. D. Miller, Appl. Phys. Lett. 103, 033503 (2013).

${ }^{25}$ J. Fabiańska, G. Kassier, and T. Feurer, Sci. Rep. 4, 5645 (2014).

${ }^{26}$ F. O. Kirchner, A. Gliserin, F. Krausz, and P. Baum, Nat. Photonics 8, 52 (2014).

${ }^{27}$ C. Kealhofer, W. Schneider, D. Ehberger, A. Ryabov, F. Krausz, and P. Baum, Science 352, 429 (2016).

${ }^{28}$ D. J. Bradley and G. H. New, Proc. IEEE 62, 313 (1974).

${ }^{29}$ G. Mourou and W. Knox, Appl. Phys. Lett. 36, 623 (1980).

${ }^{30}$ C. H. Lee, Appl. Phys. Lett. 30, 84 (1977).

${ }^{31}$ W. Knox and G. Mourou, Opt. Commun. 37, 203 (1981)

${ }^{32}$ V. Guidi and A. V. Novokhatsky, Meas. Sci. Technol. 6, 1555 (1995). 
${ }^{33}$ Z. Chang, A. Rundquist, J. Zhou, M. M. Murnane, H. C. Kapteyn, X. Liu, B. Shan et al., Appl. Phys. Lett. 69, 133 (1996).

${ }^{34}$ A. Maksimchuk, M. Kim, J. Workman, G. Korn, J. Squier, D. Du, D. Umstadter, G. Mourou, and M. Bouvier, Rev. Sci. Instrum. 67, 697 (1996).

${ }^{35}$ J. Larsson, Z. Chang, E. Judd, P. J. Schuck, R. W. Falcone, P. A. Heimann, H. A. Padmore et al., Opt. Lett. 22, 1012 (1997).

${ }^{36}$ D. X. Wang, G. A. Krafft, and C. K. Sinclair, Phys. Rev. E 57, 2283 (1998).

${ }^{37}$ A. V. Aleksandrov, N. S. Dikansky, V. Guidi, G. V. Lamanna, P. V. Logatchov, S. V. Shiyankov, and L. Tecchio, Rev. Sci. Instrum. 70, 2622 (1999).
${ }^{38}$ C. Belzile, J. C. Kieffer, C. Y. Cote, T. Oksenhendler, and D. Kaplan, Rev. Sci. Instrum. 73, 1617 (2002).

${ }^{39}$ J. Liu, J. Wang, B. Shan, C. Wang, and Z. Chang, Appl. Phys. Lett. 82, 3553 (2003).

${ }^{40}$ M. M. Shakya and Z. Chang, Proc. SPIE 5534, 125-131 (2004).

${ }^{41}$ O. J. Luiten, S. B. Van der Geer, M. J. De Loos, F. B. Kiewiet, and M. J. Van Der Wiel, Phys. Rev. Lett. 93(9), 094802 (2004).

${ }^{42}$ C. H. Lee, Picosecond Optoelectronic Devices (Academic, Orlando, 1984), p. 378.

${ }^{43}$ H. R. Philipp and H. Ehrenreich, Phys. Rev. 129, 1550 (1963).

${ }^{44}$ M. Schall and P. U. Jepsen, Opt. Lett. 25, 13 (2000). 